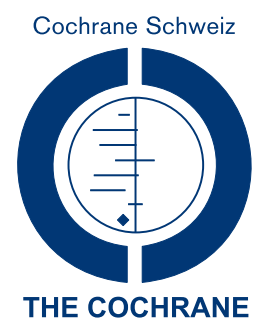

COLLABORATION ${ }^{\circledR}$

Institut universitaire de médecine sociale et préventive (CHUV et Université de Lausanne) et Cochrane Suisse'; Département de gynécologie obstétrique ${ }^{2}$, Service d'anesthésiologie, Sécteur maternité3, CHUV Lausanne

'M. Rège-Walther, ${ }^{2} G$. Théry, ${ }^{3}$ L. Thierrin, 'I. Peytremann-Bridevaux

\title{
Sollen Gebärende essen und trinken dürfen?
}

\section{Bibliographie}

Singata M, Tranmer J, Gyte GML: Restricting oral fluid and food intake during labour. Cochrane Database of Systematic Reviews 2010, Issue 1. Art. No.: CDoo3930.

DOI:1002/14651858.CDoo3930.pub2.
Diese Rubrik gibt die Resultate einer systematischen Review wieder, die durch die Cochrane Collaboration in der Cochrane Library (www.cochrane.org/) publiziert wurde. Der Artikel beschränkt sich auf eine klar umschriebene Fragestellung und fasst den aktuellen Kenntnisstand dazu zusammen. Es handelt sich deshalb nicht um eine Leitlinie (Guideline) zur Behandlung einer bestimmten Erkrankung.

Der Artikel ist in französischer Sprache in der Revue Médicale Suisse erschienen.

Rev Med Suisse 2010; 6: 1984.

\section{Fallvignette}

Zwei Ihrer Patientinnen befinden sich im Entbindungssaal und würden gerne etwas essen und/oder trinken. Die erste Patientin ist eine 32-jährige Frau in guter gesundheitlicher Verfassung am Anfang der Entbindung (zweite normal ausgetragene Schwangerschaft), bei der eine Periduralanästhesie gesetzt wurde. Die zweite Patientin ist eine 27-jährige Frau am Beginn des Geburtsvorgangs (erste ausgetragene Schwangerschaft), bei der eine Kontraindikation gegenüber einer Periduralanästhesie besteht. Sie ist übergewichtig und die Untersuchung der Mundhöhle lässt eine schwierige Intubation vermuten. Zudem ist der Herzrhythmus des Fötus auffällig.

\section{Frage}

Welche Empfehlungen geben Sie Ihren Patientinnen betreffend Nahrungsaufnahme während der Entbindung?

\section{Hintergrund}

Seit über 60 Jahren ist es in den meisten Entbindungsstationen gängige Praxis, Frauen während des Geburtsvorgangs den Konsum von Getränken oder Speisen teilweise oder ganz zu untersagen. Diese Praxis ist jedoch regional unterschiedlich und ändert sich immer wieder. Das Ziel dieser systematischen Review besteht darin, bei Frauen mit geringem
Risiko für eine Vollnarkose zu beurteilen, welche Vor- oder Nachteile mit dem Konsum von Getränken und/oder Speisen während der Entbindung verbunden sind, und zwar sowohl für die Frauen als auch für die Neugeborenen.

\section{Resultate}

In der Metaanalyse von fünf randomisierten Vergleichsstudien mit insgesamt 3130 Frauen wurde Trinken oder Essen mit nüchternen Zustand oder dem Lutschen von Eiswürfeln während des Gebärens verglichen:

- Das Risiko für einen Kaiserschnitt war nicht erhöht (fünf Studien; RR: 0,9; 95\% CI: 0,6-1,3).

- Das Risiko für eine assistierte Entbindung (Saugglocke oder Geburtszange bei vaginaler Geburt) war nicht erhöht (fünf Studien; RR: 0,98; 95\% CI: 0,9-1,1).

- Die Dauer der Geburt war nicht verlängert (drei Studien; MD: -0,3; 95\% CI: -1,6-0,97).

- Das Risiko für Erbrechen war nicht erhöht (drei Studien; RR: 0,9; 95\% CI: $0,6-1,3)$.

- Beim Neugeborenen war das Risiko, nach 5 Minuten einen Apgar-Score $<7$ zu haben, nicht erhöht (vier Studien RR: 1,$4 ; 95 \%$ CI: 0,8-2,7).

\section{Einschränkungen}

- Aufgrund der Anzahl der eingeschlossenen Teilnehmerinnen fällt eine Studie gegenüber den anderen stark ins Gewicht. 
- Die Ergebnisse lassen sich nicht auf schwangere Frauen mit einem höheren Risiko für eine Vollnarkose übertragen.

- Das Mendelson-Syndrom (Aspiration während/nach der Narkose) konnte nicht untersucht werden, da es bei keiner Frau auftrat.

- Wichtige Aspekte wie die Zufriedenheit der Mütter oder das Auftreten von Hypoglykämien beim Neugeborenen wurden nicht erfasst.

\section{Schlussfolgerung der Autoren}

Trinken und/oder Essen während der Entbindung ist bei geringem Komplikationsrisiko weder für die Mutter noch für das Neugeborene mit einem besonderen Vorteil oder einer besonderen Gefahr verbunden. Die Wahl, etwas zu sich zu nehmen, sollte daher den Frauen selbst überlassen werden.
Ob ein Ess-/Trinkverbot bei Frauen mit erhöhtem Risiko für eine Vollnarkosesinnvoll ist, lässt sich nicht abschliessend beurteilen, da für sie noch keine Ergebnisse aus randomisierten Vergleichsstudien vorliegen.

\section{Antwort auf die Frage}

In Abstimmung mit dem üblichen Vorgehen in Ihrer Einrichtung können Sie der Patientin eine leichte Kost empfehlen, solange kein besonderes Risiko vorliegt.

Hingegen sollten Sie einer RisikoPatientin ganz von fester Nahrung abraten und ihr die Gründe erklären. Allerdings steht ihr die Wahl trotzdem frei.
Redaktionelle Koordination Prof. Dr. med. B. Burnand (MPH),

Dr. med. I. Peytremann-Bridevaux (MPH), M. Rège Walther (MSc, MA),

Dr. med. E. von Elm (MSc)

Cochrane Suisse, Institut universitaire de médecine sociale et préventive, Biopôle 2 , Corniche 10, 1010 Lausanne swiss.cochrane@chuv.ch

\section{Korrespondenzadresse}

M. Rège-Walther

Cochrane Suisse

Institut universitaire de médecine sociale et préventive

CHUV et Université de Lausanne

Biopôle 2

Corniche 10

1010 Lausanne

swiss.cochrane@chuv.ch

\section{Kostenloser Zugang zur Cochrane Library}

Die Schweizerische Akademie der medizinischen Wissenschaften (SAMW) hat eine Initiative gestartet, um niedergelassenen Ärzten/innen einen besseren Zugang zur medizinischen Fachliteratur zu ermöglichen. Unter anderem ermöglicht sie einen kostenfreien Zugang zur Cochrane Library. Ab sofort können sich die Ärzte dafür bei der SAMW registrieren.

Details unter http://www.samw.ch/de/Projekte/Fachliteratur.html 